\title{
Article \\ Multi-Objective Optimization of Permanent Magnet Synchronous Motor for Electric Vehicle Considering Demagnetization
}

\author{
Yong-min You ${ }^{1}\left[\right.$ and Keun-young Yoon ${ }^{2, *}$ \\ 1 Department of Automotive Engineering, Honam University, 417 Eodeung-daero, Gwangsan-gu, \\ Gwangju 62399, Korea; ym.you@honam.ac.kr \\ 2 Department of Electrical Engineering, Honam University, 417 Eodeung-daero, Gwangsan-gu, \\ Gwangju 62399, Korea \\ * Correspondence: ky.yoon@honam.ac.kr; Tel.: +82-62-940-5489
}

Citation: You, Y.-m.; Yoon, K.-y. Multi-Objective Optimization of Permanent Magnet Synchronous Motor for Electric Vehicle

Considering Demagnetization. Appl. Sci. 2021, 11, 2159. https://doi.org/ 10.3390/app11052159

Received: 25 January 2021

Accepted: 22 February 2021

Published: 1 March 2021

Publisher's Note: MDPI stays neutral with regard to jurisdictional claims in published maps and institutional affiliations.

Copyright: (C) 2021 by the authors. Licensee MDPI, Basel, Switzerland. This article is an open access article distributed under the terms and conditions of the Creative Commons Attribution (CC BY) license (https:// creativecommons.org/licenses/by/ $4.0 /)$.
Abstract: The irreversible demagnetization of permanent magnets causes the deterioration of the performance in permanent magnet synchronous motors (PMSMs), which are used for electric vehicles. $\mathrm{NdFeB}$, which is the permanent magnet most commonly used in PMSMs for electric vehicles, is easily demagnetized at high temperatures. Because traction motors for electric vehicles reach high temperatures, and a high current can be instantaneously applied, permanent magnets of PMSM can be easily demagnetized. Therefore, it is important to study the demagnetization phenomenon of PMSMs for electric vehicles. However, since the demagnetization analysis procedure is complicated, previous studies have not been able to perform optimization considering demagnetization characteristics. In this study, we optimized the shape of a PMSM for electric vehicles by considering the demagnetization characteristics of permanent magnets using an automated design of experiments procedure. Using this procedure, a finite element analysis for each experimental point determined by a sampling method can be performed quickly and easily. The multi-objective function minimizes the demagnetization rate and maximizes the average torque, and the constraints are the efficiency and torque ripple. Various metamodels were generated for each of the multi-objective functions and constraints, and the metamodels with the best prediction performance were selected. By applying a multi-objective genetic algorithm, 1902 various optimal solutions were obtained. When the weight rate of the demagnetization rate to the torque was set to $0.1: 0.9$, the demagnetization rate and average torque were improved by $4.45 \%$ and $2.7 \%$, respectively, compared to those of the initial model. The proposed multi-objective optimization method can guide the design of PMSMs for electric vehicles with high reliability and strong demagnetization characteristics.

Keywords: optimization; PMSM; electric vehicle; demagnetization; metamodel; MOGA

\section{Introduction}

As traction motors for electric vehicles, permanent magnet synchronous motors (PMSMs) have been widely used owing to their high power density and efficiency. In most PMSMs, NdFeB series magnets are used. Although these magnets have high residual magnetic flux density and coercivity, they have the disadvantage of being easily demagnetized at high temperatures. When a permanent magnet is demagnetized irreversibly, the motor performances, such as the torque and efficiency, are deteriorated. This is particularly problematic because traction motors for electric vehicles work at high temperatures and high currents, so the permanent magnet is easily demagnetized. Therefore, it is important to study the demagnetization phenomenon of PMSMs for electric vehicles.

Many studies have been conducted on the demagnetization of PMSMs. In [1], the demagnetization characteristic of a PMSM with dysprosium-free permanent magnets was studied. The demagnetization of the permanent magnets was examined after applying a demagnetization current three times higher than the rated current at $150{ }^{\circ} \mathrm{C}$. The magnetic flux density distribution of the permanent magnets was converted into a temperature to 
determine where the permanent magnets were demagnetized. In [2], the demagnetization of $45 \mathrm{SH}$ grade permanent magnets was examined after applying a demagnetization current three, five, and seven times higher than the rated current at a temperature of $100{ }^{\circ} \mathrm{C}$. Changes in the magnetic flux density distribution and demagnetization rate according to the separated number of permanent magnets were examined. Other studies examined the change in the back electromotive force (EMF) and magnetic flux density distribution of a motor after applying a demagnetization current one and a half to four times higher than the rated current at temperatures of $100-160{ }^{\circ} \mathrm{C}$ [3-5]. Although the demagnetization of permanent magnets according to the temperature and current was partially analyzed, the optimal design of motors considering the demagnetization characteristics was not achieved. The reason that the demagnetization characteristics were not considered in the optimal design is that the analysis process is complicated. For optimal design, computational analysis of many experimental points must be performed. However, it is not easy to perform such analyses at each experimental point and use the results for optimal design. Moreover, it is almost impossible to consider demagnetization characteristics in the optimal design performed manually.

On the other hand, many studies have been conducted on the optimal design with multi-objective functions [5-7]. For such designs, the weights of the multi-objective functions affect the optimal design results. However, in most previous studies, optimization was performed with fixed weights. In the case of using multi-objective functions, the optimal design result according to the change in weight should be carefully reviewed [8].

As an optimal design method, the use of metamodels is widely used. After computational analyses or experiments are performed as many times as the number of design of experiments (DOE), metamodels for objective functions and constraints are established based on the results. The generated metamodels are in the form of approximated functions and are combined with an optimization algorithm to find an optimal solution to a design problem. Since there are various types of metamodel techniques for optimal design, it is important to apply a proper metamodel technique with high accuracy according to each design problem [9]. However, in most of the previous studies, optimization was usually performed using only one metamodel technique, e.g., the response surface method or Kriging [10-16].

The distinguishing points of this paper from the previous research cases mentioned above are as follows. First, we optimized the PMSM design considering the demagnetization characteristics of the permanent magnets. The previous studies have not considered demagnetization characteristics as objective functions or constraints of the optimal design because the analysis process is complex. However, in this study, the demagnetization characteristic was set as the objective function of the optimal design by using an automated linkage analysis procedure with a finite element analysis (FEA) software and an optimal design software [9]. Second, the effects of the weight of the multi-objective function on the optimal design result were carefully examined by applying the multi-objective genetic algorithm (MOGA). Therefore, it was possible to select the desired optimal design result according to the user's purpose or application. Furthermore, we approximated the multiobjective functions and constraints using various metamodeling techniques. The accuracy of the optimal design result was ensured by performing optimal design using metamodels with the best prediction performance.

In this study, a multi-objective optimization of a PMSM for electric vehicles was conducted considering the demagnetization characteristics. A multi-objective function was established to maximize the average torque and minimize the demagnetization rate. As constraints, the torque ripple and efficiency were selected. Five design variables were selected to satisfy the multi-objective function and constraints. Fifty experimental points were determined through the optimal Latin hypercube design (OLHD) [17]. Design of experiments (DOE) for 50 experimental points was carried out through an automated linkage analysis procedure using an FEA software and an optimal design software [9]. From the DOE results, we examined the correlation between design variables and responses 
using a screening technique. Metamodels of multi-objective functions and constraints were established using the DOE results, and the metamodels with the highest accuracy were selected through accuracy evaluation. These metamodels were combined with the MOGA to find various optimal solutions according to the weight [18].

\section{Demagnetization Analysis}

The demagnetization characteristics of PMSMs for electric vehicles are important. The irreversible demagnetization of the permanent magnets in PMSMs causes the degradation in the performance of the motors. Manufacturers have to control the demagnetization characteristics of PMSMs for electric vehicles carefully [1]. To prevent demagnetization, General Motors controls the $\mathrm{BH}$ characteristics of permanent magnets at $140^{\circ} \mathrm{C}$. Hyundai Motor Company and LG Electronics are also managing to prevent demagnetization of traction motors at $150{ }^{\circ} \mathrm{C}$.

\subsection{Initial Model}

The shape and specifications of the initial model are shown in Figure 1 and Table 1, respectively. The PMSM used in this study had 8 poles and 36 slots, and NdFeB magnets of N39UH grade were applied. The permanent magnets were arranged in a V-shape to concentrate the magnetic flux. The armature winding was wound in a distributed form for harmonic reduction.

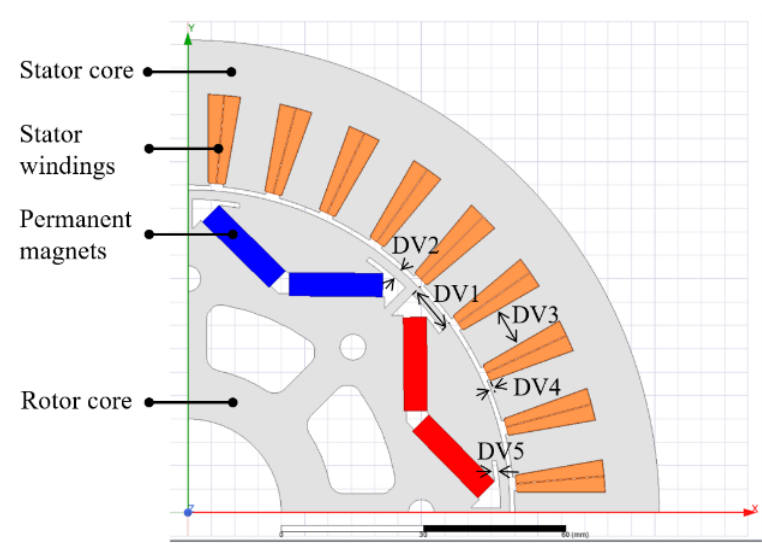

Figure 1. Shape of the initial model.

Table 1. Specifications of the initial model.

\begin{tabular}{cccc}
\hline & Items & Unit & Value \\
\hline \multirow{3}{*}{ Target } & Maximum output power & $\mathrm{kW}$ & 15 \\
& Maximum torque & $\mathrm{N} \cdot \mathrm{m}$ & 60 \\
& Rated speed & $\mathrm{rpm}$ & 2387 \\
\hline \multirow{2}{*}{ Material } & Permanent magnet & - & 35PN210 (POSCO) \\
& Electrical steel & - & N39UH (ShinEtsu) \\
\hline \multirow{3}{*}{ Electrical } & Continuous current & $\mathrm{A}_{\mathrm{rms}}$ & 146 \\
& Current phase angle & $\circ$ & 25 \\
& Phase resistance & $\Omega$ & 0.021 \\
\hline \multirow{3}{*}{ Mechanical } & Stator outer diameter & $\mathrm{mm}$ & 202 \\
& Rotor outer diameter & $\mathrm{mm}$ & 138 \\
& Air-gap length & $\mathrm{mm}$ & 1 \\
& Lamination length & $\mathrm{mm}$ & 45 \\
\hline \multirow{2}{*}{ Thermal } & Reference temperature & ${ }^{\circ} \mathrm{C}$ & 20 \\
\cline { 2 - 4 } & Demagnetization temperature & ${ }^{\circ} \mathrm{C}$ & 150 \\
\hline
\end{tabular}




\subsection{Demagnetization Analysis Conditions}

The demagnetization characteristics of permanent magnets depend on the temperature. Figure 2 shows the demagnetization curves of ShinEtsu N39UH grade magnets. It can be seen that demagnetization occurs more easily at higher temperatures. The demagnetization analysis was performed while applying a demagnetization current 5, 6, and 7 times higher than the rated current at $160^{\circ} \mathrm{C}$. The demagnetization analysis was performed at $2387 \mathrm{rpm}$ because the current applied in this region was the largest. For the demagnetization analysis, the electromagnetic field analysis software Ansys Maxwell was utilized. The demagnetization current applied to the $U$ phase is calculated as follows [19]:

$$
\text { if }\left(t<\left(\frac{\frac{1}{r p s}}{4}\right), 0, \text { if }\left(t>\left(\frac{\frac{2}{r p s}}{4}\right), 0, I_{\text {demag }}\right)\right) * \sin (\omega t+\beta)
$$

where $t$ is the time, $r p s$ is the revolution per second, $I_{\text {demag }}$ is the demagnetization current, $\omega$ is the angular velocity, and $\beta$ is the current phase angle. Figure 3 shows the current waveforms in the $\mathrm{U}, \mathrm{V}$, and $\mathrm{W}$ phases while applying a demagnetization current 5 times higher than the rated current.

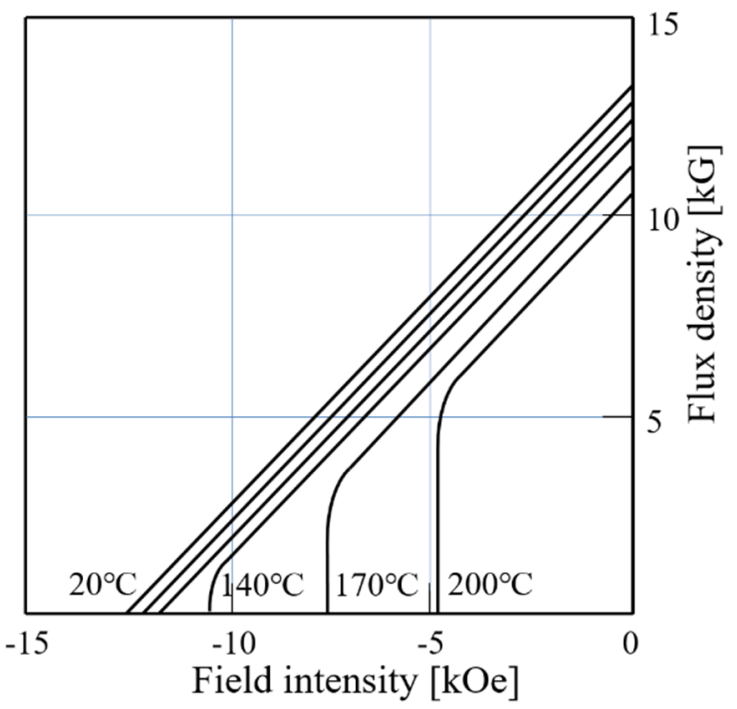

Figure 2. Demagnetization curves of N39UH grade magnets.

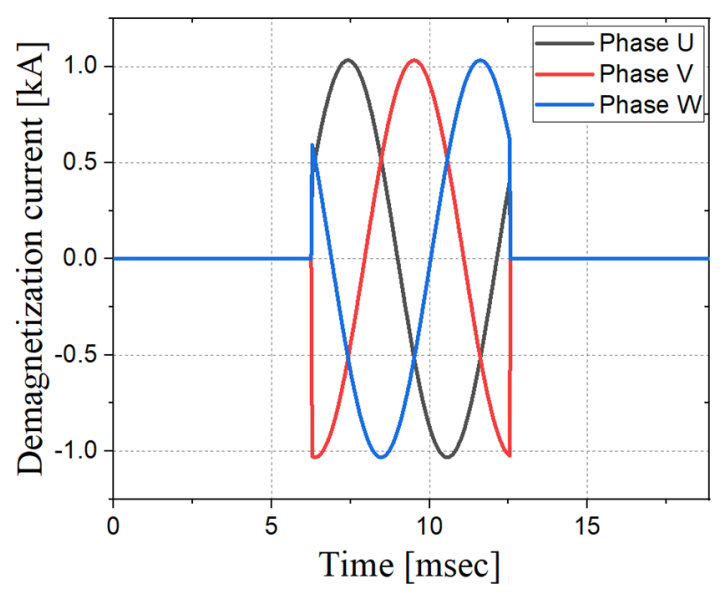

Figure 3. Demagnetization current waveforms. 


\subsection{Demagnetization Analysis Results}

The demagnetization rate can be determined by checking the magnetic field strength after applying the demagnetization current. However, because the magnetic field strength cannot be measured in an actual manufacturing line, the demagnetization rate is generally determined based on the change in back EMF after applying the demagnetization current. In addition, in order to carry out a large number of designs of experiments, the demagnetization rate should be defined as a value that can be easily quantified; so, the demagnetization rate was set based on the change in the back EMF. Figure 4 shows the back EMF waveform after a demagnetization current 5, 6, and 7 times higher than the rated current is applied. The demagnetization rate is defined as the rate of change of the back EMF after demagnetization and is calculated as follows [2,19]:

$$
\text { Demag_ratio }=\frac{B E M F_{i n i}-B E M F_{\text {demag }}}{B E M F_{\text {ini }}} \times 100[\%]
$$

where Demag_ratio is the demagnetization ratio, $B E M F_{i n i}$ is the initial value of the back $E M F$, and $B E M F_{\text {demag }}$ is the value of the back EMF after demagnetization. When the demagnetization current is 5,6 , and 7 times higher than the rated current, the demagnetization rates are $1.7 \%, 6.4 \%$, and $13.7 \%$, respectively. Figure 5 shows the distribution of the magnetic flux density of the permanent magnets after the demagnetization. It can be seen that partial demagnetization occurs at the edge of the permanent magnets.

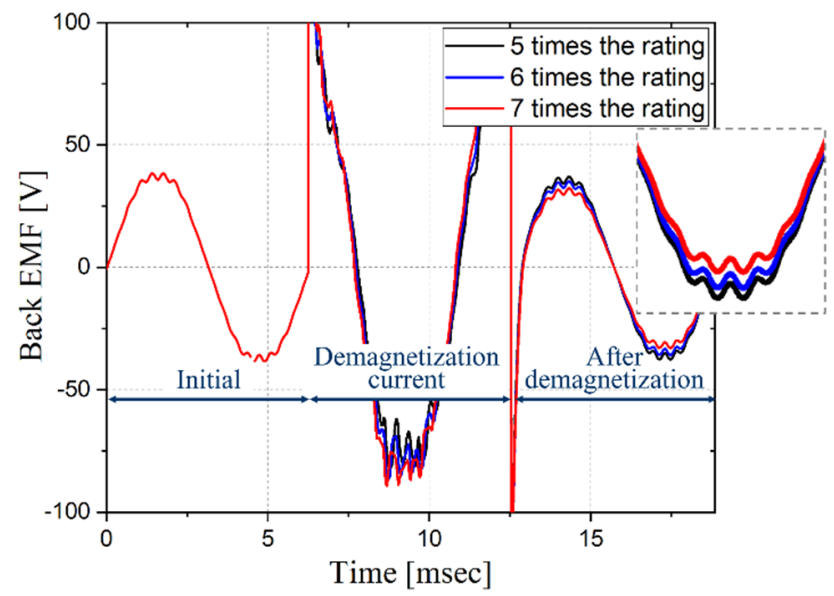

Figure 4. Back electromotive force (EMF) waveforms after the demagnetization.

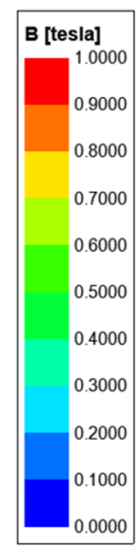

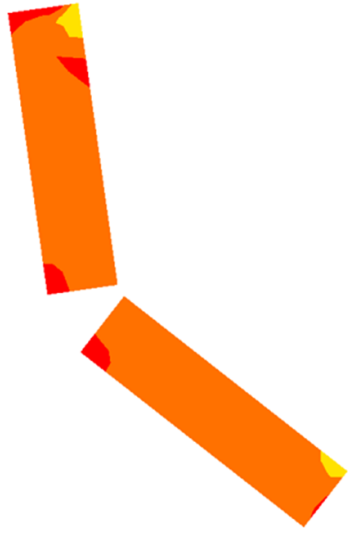

(a)

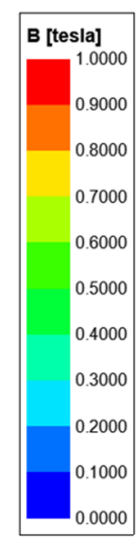

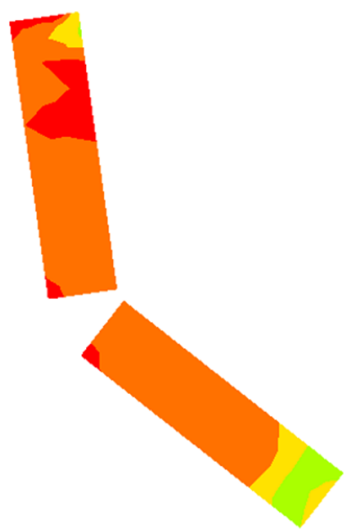

(b)
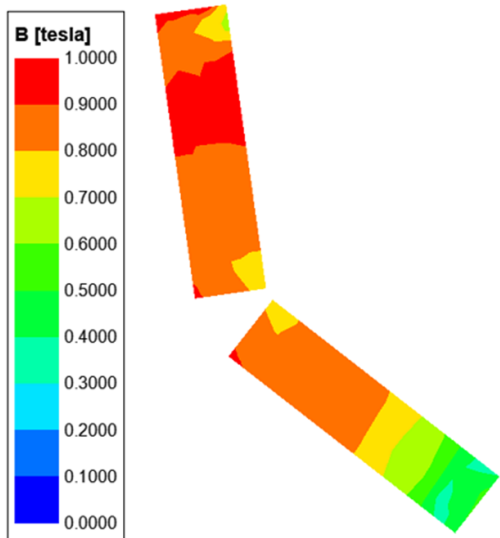

(c)

Figure 5. Magnetic flux density of the permanent magnets after the demagnetization: (a) 5 times, (b) 6 times, and (c) 7 times higher than the rated current. 


\section{Design Optimization}

\subsection{Optimization Process}

Figure 6 shows the shape optimization process of the PMSM to minimize the demagnetization rate of the permanent magnets and maximize the average torque. First, the multi-objective functions, constraints, and optimal design variables were established. Next, an initial modeling and parametric analysis setting for the automatic change of design variables was performed using the FEA software Maxwell. Next, DOE was executed using an automated DOE procedure through co-simulation between Maxwell and the optimal design software PIAnO, which uses Maxwell's macro functions [9]. By creating VBSript files and batch files, load analysis and demagnetization analysis were automatically performed in Maxwell. Maxwell's analysis results were transferred to PIAnO, and the responses of multi-objective functions and constraints were automatically calculated. In addition, when the computational analysis for one experimental point was finished, the shape design parameters were automatically changed in Maxwell without the aid of CAD tools by receiving PIAnO's command. This process was automatically repeated as many times as the number of DOE.

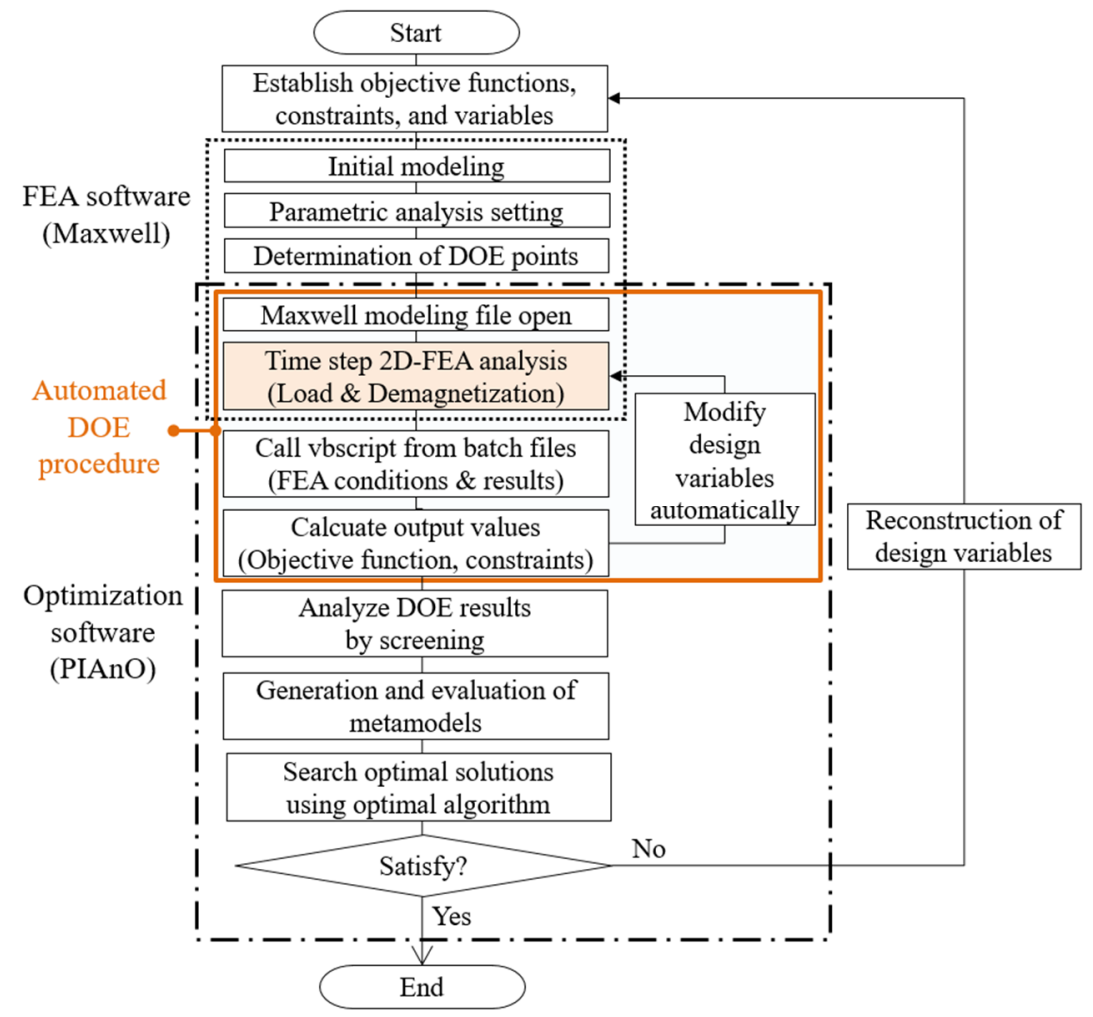

Figure 6. Optimization design process.

To increase the accuracy of the metamodels, it was necessary to obtain as many DOE results as possible. However, in most previous studies, DOE was performed manually and an insufficient amount of data was obtained. Furthermore, because demagnetization analyses are complex, they were not included in the DOE [1-5].

In this study, the demagnetization analysis was included in the DOE process using the automated DOE procedure. From the obtained DOE results, the influence of design variables on the multi-objective functions and constraints was analyzed through a screening technique. Next, metamodels of multi-objective functions and constraints were established using the DOE results, and the best metamodels were selected through accuracy evaluation. Finally, metamodels were combined with an optimal algorithm to obtain optimal solutions. 


\subsection{Multi-Objective Function, Constraints, and Design Variables}

The multi-objective function should minimize the demagnetization rate and maximize the average torque as follows:

$$
\text { Minimize } F_{o b j}=\frac{\text { Demag_ratio }}{T_{a v e} e^{w 2}}
$$

where $F_{o b j}$ is the multi-objective function, $T_{a v e}$ is the average value of the torque, and $w 1$ and $w 2$ are the weight coefficients. The efficiency and torque ripple were selected as constraints. The efficiency was set to $91.55 \%$ or more based on the value of the initial model, and the torque ripple was set to less than $10 \%$, which is the level allowed in the traction motor for electric vehicles [20]. The design variables to satisfy the multi-objective function and constraints are shown in Figure 1, and the range of the design variables is shown in Table 2 [8]. The size of the permanent magnet has a great influence on the demagnetization properties. The larger the size of the permanent magnet, the better the demagnetization property, but the material cost will also increase. Therefore, the size of the permanent magnet is not generally considered as an optimal design variable.

Table 2. Mechanical constraints of the design variables.

\begin{tabular}{ccccc}
\hline Design Variables & Unit & Lower Limit & Upper Limit & Considerations \\
\hline Barrier length & $\mathrm{mm}$ & -4 & 10 & Magnetic flux flow \\
Rib thickness & $\mathrm{mm}$ & -1 & 0.5 & Mechanical stiffness and interference \\
Teeth width & $\mathrm{mm}$ & -1 & 0 & Coil fill factor and magnetic saturation \\
Teeth thickness & $\mathrm{mm}$ & 0 & 1 & Mechanical interference and magnet flux flow \\
Barrier gap & $\mathrm{mm}$ & 0 & 2 & \\
\hline
\end{tabular}

\subsection{Design of Experiment}

The OLHD technique, which is a DOE sampling technique suitable for computational experiments, was used in this study. OLHD can improve the space filling characteristics by using optimal conditions and distributing the test points evenly [17]. Considering the fact that there are five design variables, the number of experiments was determined to be 50 [9]. The best experimental point was obtained through analysis for 50 experimental points, and the comparison results with the initial model are shown in Table 3. The demagnetization rate of the best experimental point was $2.466 \%$, which is very low compared to that of the initial model. However, the efficiency and average torque of the best point is slightly lower than those of the initial model. Figure 7 shows the screening analysis result. It can be seen that the average torque, torque ripple, and efficiency are most affected by the change in barrier length, and the demagnetization rate is most affected by the change in rip thickness.

Table 3. Comparison results between the initial model and the best experimental point.

\begin{tabular}{cccc}
\hline Design Variables & Unit & Initial Model & Best Point \\
\hline Barrier length & $\mathrm{mm}$ & 0 & -3.72 \\
Rib thickness & $\mathrm{mm}$ & 0 & 0.07 \\
Teeth width & $\mathrm{mm}$ & 0 & 0.44 \\
Teeth thickness & $\mathrm{mm}$ & 0 & 0.62 \\
Barrier gap & $\mathrm{mm}$ & 0 & 1.36 \\
Demagnetization rate & $\%$ & 6.397 & 2.466 \\
Average torque & $\mathrm{N} \cdot \mathrm{m}$ & 60.936 & 59.846 \\
Torque ripple & $\%$ & 5.202 & 4.939 \\
Efficiency & $\%$ & 91.550 & 91.398 \\
\hline
\end{tabular}




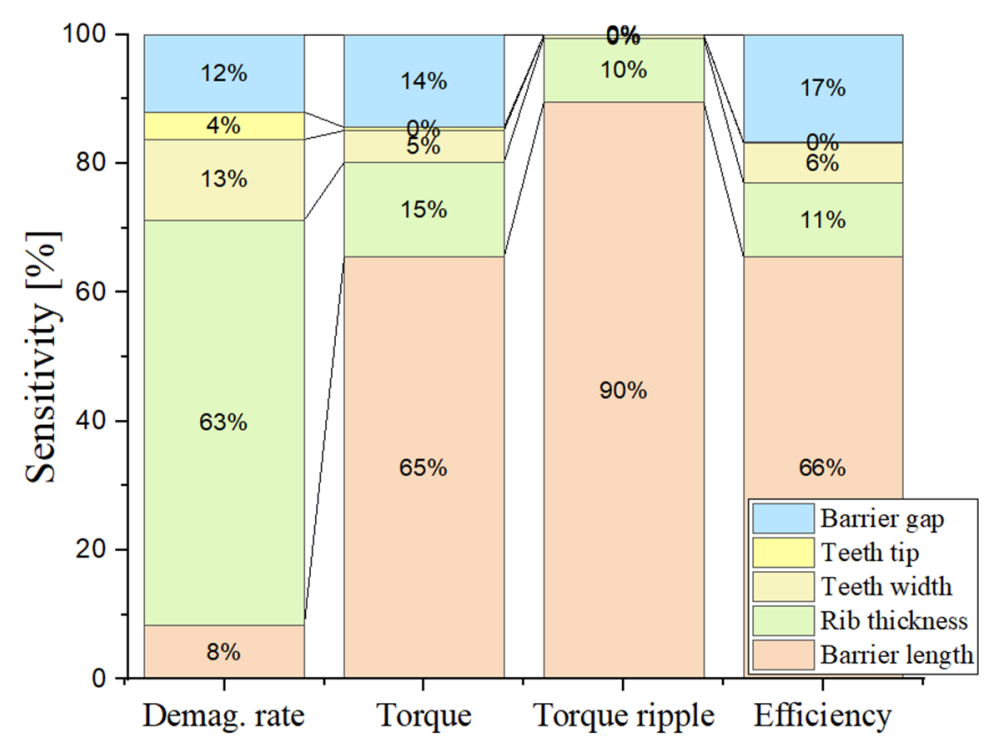

Figure 7. Sensitivity analysis using screening.

\subsection{Metamodeling}

To optimize the motor shape using the DOE results, metamodels must be established. Metamodeling is the process of approximating the DOE results in the form of a function. Although different metamodeling techniques could be used for different design problems, in previous studies, metamodels of objective functions and constraints were established by applying a single metamodeling technique [10-16]. In this study, we established metamodels by applying 13 techniques for each multi-objective function and constraint, and the most accurate technique was selected through accuracy evaluation. The accuracy of the metamodels was evaluated in terms of the root mean squared error (RMSE) [21]:

$$
\text { RMSE }=\sqrt{\frac{1}{n E X P_{-} \text {ts }} \sum_{i=1}^{\text {nEXP_ts }}\left[y\left(X_{i}\right)-\hat{y}\left(X_{i}\right)\right]^{2}}
$$

where nEXP_ts is the number of test points, $y\left(X_{i}\right)$ is the real function value, and $\hat{y}\left(X_{i}\right)$ is the metamodel value. Table 4 shows the results of the accuracy evaluation of the metamodels. It can be seen that when the custom multi-layer perceptron (MLP) technique, which is a deep learning algorithm, is applied, the accuracy of the metamodel is the best for the demagnetization rate and torque. When the simple cubic polynomial regression (PRG) technique and the Krigging technique are applied to the efficiency and torque ripple, the metamodel has the highest accuracy.

Neural networks are complex structures made up of artificial neurons that can take multiple inputs and produce a single output. Neurons transform data mathematically, and neural networks can use these neurons to learn complex patterns. Neurons are mathematical functions, also known as perceptrons. One or more inputs are multiplied by weights and then passed the result to a nonlinear function called the activation function. A layer made up of multiple neurons is called a hidden layer. The MLP has been used for the shape optimization of electric devices [22]. MLP is a type of feedforward artificial neural network and has the advantage of being able to represent a nonlinear relationship between input and output variables. The metamodels of the demagnetization rate and torque are generated using the MLP technique. Various hyperparameters required to apply the MLP technique are optimized through the hyperparameter optimization function of PIAnO [21]. The number of hidden layers of the demagnetization rate is 2 , and the number of neurons in each layer is 178 and 2. The number of hidden layers of the torque is 4 , and the number of neurons in each layer ranges from 8 to 178. A rectified linear unit is used as the activation function of demagnetization rate and torque. 
Table 4. Root mean squared error (RMSE) test results of metamodels.

\begin{tabular}{ccccc}
\hline Metamodel & Demanetizaion Rate & Torque & Torque Ripple & Efficiency \\
\hline Krigging & 0.299578388 & 0.480370464 & 0.661102263 & 0.068150470 \\
RBF (Interpolation) & 0.162832835 & 0.316088471 & 1.684960769 & 0.054181548 \\
RBF (Regression) & 0.184083130 & 0.185910892 & 1.660534066 & 0.049919916 \\
PRG (Forward step) & 0.219662647 & 0.199215669 & 1.649310197 & 0.054889271 \\
PRG (Backward step) & 0.245157992 & 0.365451547 & 1.344382800 & 0.041693768 \\
PRG (Linear) & 0.281644165 & 0.811042784 & 1.465072536 & 0.105565885 \\
PRG (Simple quadratic) & 0.249900294 & 0.907408134 & 1.366168145 & 0.126547557 \\
PRG (Full quadratic) & 0.194996813 & 0.197566341 & 1.847404658 & 0.037366471 \\
PRG (Simple cubic) & 0.195295038 & 0.221251507 & 1.524009121 & 0.034044144 \\
EDT (Hybrid) & 0.563395063 & 0.855283102 & 1.071512674 & 0.116880624 \\
EDT (Hybrid / opt.) & 0.486268030 & 0.767061634 & 1.265285952 & 0.094801174 \\
MLP & 0.252059717 & 1.833024299 & 1.687341801 & 0.168041319 \\
MLP (Custom) & 0.111563863 & 0.159207851 & 1.861550458 & 0.114412812 \\
\hline
\end{tabular}

\subsection{Optimization Results}

The MOGA is a global optimization method suitable for multi-objective function problems. It uses the non-dominant sorting and crowding distance and is based on a genetic algorithm. This algorithm can be used without encoding the design variables if actual values are used, making calculations simpler and allowing continuous and discrete design variables to be processed simultaneously [18]. Most previous studies on the optimal design with multi-objective functions found optimal solutions with fixed weights, making it difficult to determine the influence of the individual weights on the optimal result. This influence can be easily determined with the MOGA.

In this study, the metamodels of the multi-objective functions and constraints with the best accuracy were combined with the MOGA to find optimal solutions. We used a workstation with an Intel Xeon Silver 4110 processor (11 M cache, $2.1 \mathrm{GHz}$ ) and $64 \mathrm{~GB}$ of RAM. The number of function calls was 25,002, and the optimization time was $6 \mathrm{~min}$ and $44 \mathrm{~s}$. Figure 8 shows the optimization results for the demagnetization rate and average torque. The blue dot represents the initial model value, and the gray dots represent 25,002 analysis solutions in the optimization process; the red dots represent 1902 optimal solutions obtained through optimization. From the figure, it can be seen that the 1902 solutions can be used depending on the user's needs or application.

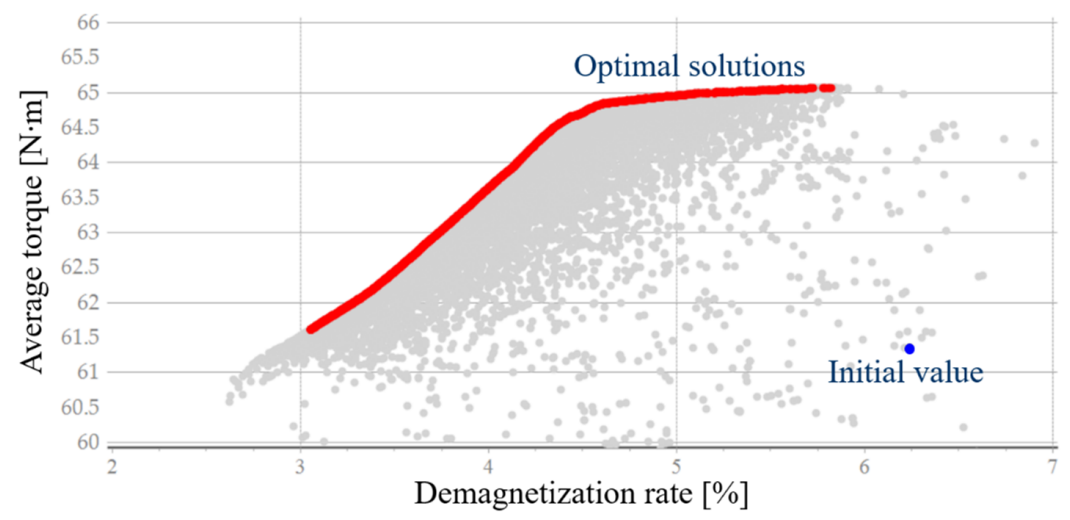

Figure 8. Pareto front of the multi-objective functions.

Because there are 1902 optimal solutions, the characteristic change was examined while changing the weight of the multi-objective function, as shown in Figure 9. The multi-objective function set in this study was to minimize the demagnetization rate and maximize the torque simultaneously. The weight of the demagnetization rate to the torque was determined to be 0.1:0.9. Because the optimal design method using metamodeling uses an approximation function, it is necessary to verify the optimal design result through FEA. Table 5 presents the verification results. This table reveals that the optimization result predicted using the metamodel and the result verified by FEA are similar. 


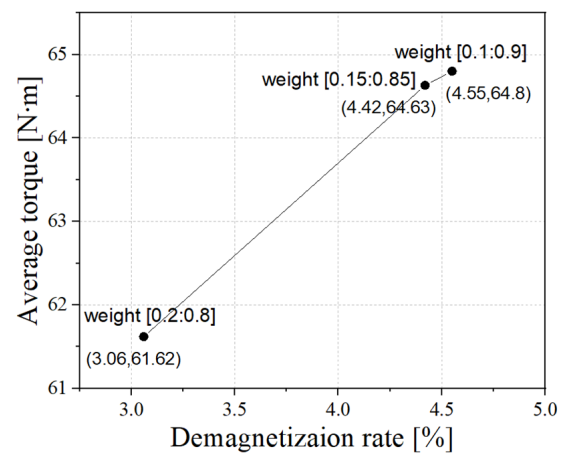

Figure 9. Change of multi-objective function according to weight change.

Table 5. Optimization results.

\begin{tabular}{|c|c|c|c|c|}
\hline Items & Unit & $\begin{array}{l}\text { Initial } \\
\text { (FEA) }\end{array}$ & $\begin{array}{c}\text { Optimal } \\
\text { (Metamodel) }\end{array}$ & $\begin{array}{c}\text { Optimal } \\
\text { (FEA) }\end{array}$ \\
\hline Barrier length & $\mathrm{mm}$ & 0 & \multicolumn{2}{|c|}{-3.999} \\
\hline Rib thickness & $\mathrm{mm}$ & 0 & \multicolumn{2}{|c|}{1.000} \\
\hline Teeth width & $\mathrm{mm}$ & 0 & \multicolumn{2}{|c|}{0.000} \\
\hline Teeth thickness & $\mathrm{mm}$ & 0 & \multicolumn{2}{|c|}{0.771} \\
\hline Barrier gap & $\mathrm{mm}$ & 0 & \multicolumn{2}{|c|}{2.000} \\
\hline Demagnetization rate & $\%$ & 6.397 & 4.554 & 3.729 \\
\hline Average torque & $\mathrm{N} \cdot \mathrm{m}$ & 60.936 & 64.795 & 63.648 \\
\hline Torque ripple & $\%$ & 5.202 & 6.299 & 7.010 \\
\hline Efficiency & $\%$ & 91.550 & 91.882 & 91.841 \\
\hline
\end{tabular}

Figure 10a shows the torque waveforms of the initial and optimal models. It can be seen that the average torque of the optimal model was $63.648 \mathrm{Nm}$, which is $4.45 \%$ higher than that of the initial model. The torque ripple of the optimal model was $7.01 \%$, which is slightly higher than that of the initial model but satisfies the constraint within $10 \%$. Figure $10 \mathrm{~b}$ shows the demagnetization rate of the initial and the optimal models. It can be seen that the demagnetization rate improved by $2.7 \%$.
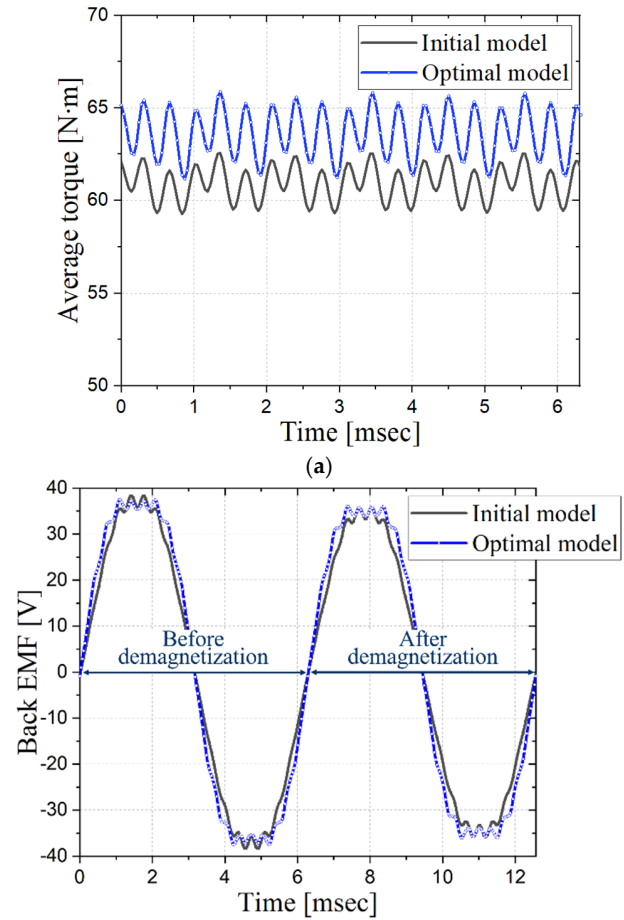

(b)

Figure 10. Torque and back EMF waveforms: (a) torque; (b) back EMF. 


\section{Conclusions}

In this study, we optimized the shape of a PMSM for electric vehicles to minimize the demagnetization rate of permanent magnets and maximize the torque of the motor. Fifty experimental points were determined by OLHD, and an automated DOE procedure combined with FEA and optimization was applied. The relationship between design variables and output responses was analyzed from the DOE results through a screening technique. Thirteen metamodels were established for the multi-objective functions and constraints, and the RMSE was evaluated as an indicator of the predictive performance of the metamodels. The metamodels with the best prediction performance were selected for each output variable, and the optimal solutions were found using the MOGA. A total of 1902 various optimal solutions were obtained. When the weight of the demagnetization rate was set to 0.1 and that of the torque was set to $0.1: 0.9$, the demagnetization rate and torque were improved by $4.45 \%$ and $2.7 \%$, respectively. Finally, the optimal design results were verified by FEA.

The significance of this study is that the optimal design was carried out in consideration of the demagnetization characteristics, which were difficult to handle, by applying the automated DOE procedure. In addition, by reviewing the application results of various metamodeling techniques, metamodels with the best predictive performance were selected and optimized. We were also able to obtain various optimal solutions by applying the MOGA suitable for multi-objective optimization. The proposed optimal design method for multi-objective functions is expected to guide the design of highly reliable traction motors for electric vehicles.

In this study, the commonly used demagnetization analysis method was applied to the optimal design, but it may differ from the actual conditions in which demagnetization occurs. In the future, we would like to conduct research on a demagnetization analysis method that is more similar to the reality. A demagnetization test was also not covered in this study because of the circumstances. Because a lot of preparation is required for a demagnetization test, we will cover it as a future research theme.

Author Contributions: Optimal design and writing, Y.-m.Y.; Analysis, writing-review and editing, K.-y.Y. All authors have read and agreed to the published version of the manuscript.

Funding: This study was supported by research fund from Honam University, 2020 and the National Research Foundation of Korea (NRF) grant funded by the Korean government (MSIT). (No. NRF2018R1C1B5046117).

Institutional Review Board Statement: Not applicable.

Informed Consent Statement: Not applicable.

Data Availability Statement: The data presented in this study are available on request from the corresponding author.

Acknowledgments: The authors express gratitude to PIDOTECH and FRONTIS for their technical support.

Conflicts of Interest: The authors declare no conflict of interest.

\section{References}

1. You, Y.-M.; Chung, D.-W. Optimal Design of a Permanent Magnet Synchronous Motor to Improve Torque and Demagnetization Characteristics. J. Magn. 2017, 22, 423-429. [CrossRef]

2. Kim, B.-C.; Lee, J.-H.; Kang, D.-W. A Study on the Effect of Eddy Current Loss and Demagnetization Characteristics of Magnet Division. IEEE Trans. Appl. Supercond. 2020, 30, 1-5. [CrossRef]

3. Zhang, Y.; McLoone, S.; Cao, W.; Qiu, F.; Gerada, C. Power Loss and Thermal Analysis of a MW High-Speed Permanent Magnet Synchronous Machine. IEEE Trans. Energy Convers. 2017, 32, 1468-1478. [CrossRef]

4. Jeong, C.-L.; Kim, Y.-K.; Hur, J. Optimized Design of PMSM With Hybrid-Type Permanent Magnet for Improving Performance and Reliability. IEEE Trans. Ind. Appl. 2019, 55, 4692-4701. [CrossRef]

5. Song, T.; Zhang, Z.; Liu, H.; Hu, W. Multi-objective optimisation design and performance comparison of permanent magnet synchronous motor for EVs based on FEA. IET Electr. Power Appl. 2019, 13, 1157-1166. [CrossRef] 
6. Guo, H.; Tian, W.; Xaiofeng, D. Multi-objective Optimal Design of Permanent Magnet Synchronous Motor for High Efficiency and High Dynamic Performance. IEEE Access 2018, 6, 23568-23581.

7. Zhao, W.; Wang, X.; Gerada, C.; Zhang, H.; Liu, C.; Wang, Y. Multi-Physics and Multi-Objective Optimization of a High Speed PMSM for High Performance Applications. IEEE Trans. Magn. 2018, 54, 1-5. [CrossRef]

8. You, Y.-M. Multi-objective optimization of a permanent magnet synchronous motor based on an automated design and analysis procedure. Microsyst. Technol. 2020, 26, 3477-3488. [CrossRef]

9. You, Y.-M. Optimal Design of PMSM Based on Automated Finite Element Analysis and Metamodeling. Energies 2019, 12, 4673. [CrossRef]

10. Kang, G.-J.; Park, C.-H.; Choi, D.-H. Metamodel-based design optimization of injection molding process variables and gates of an automotive glove box for enhancing its quality. J. Mech. Sci. Technol. 2016, 30, 1723-1732. [CrossRef]

11. Zhang, B.; Song, B.; Mao, Z.; Tian, W.; Li, B.; Li, B. A Novel Parametric Modeling Method and Optimal Design for Savonius Wind Turbines. Energies 2017, 10, 301. [CrossRef]

12. You, Y.-M.; Lipo, T.A.; Kwon, B.-I. Optimal Design of a Grid-Connected-to-Rotor Type Doubly Fed Induction Generator for Wind Turbine Systems. IEEE Trans. Magn. 2012, 48, 3124-3127. [CrossRef]

13. You, Y.M.; Jung, D.W. A Study on Performance Improvement of Polygon Mirror Scanner Motor. J. Electr. Eng. Technol. 2019, 14, 745-755. [CrossRef]

14. Kwon, J.W.; Lee, J.H.; Zhao, W.L.; Kwon, B.I. Flux-Switching Permanent Magnet Machine with Phase-Group Concentrat-ed-Coil Windings and Cogging Torque Reduction Technique. Energies 2018, 11, 2758. [CrossRef]

15. Chai, W.P.; Lipo, T.A.; Kwon, B.I. Design and Optimization of a Novel Wound Field Synchronous Machine for Torque Per-formance Enhancement. Energies 2018, 11, 2111. [CrossRef]

16. Li, Y.; Song, B.; Mao, Z.; Tian, W. Analysis and Optimization of the Electromagnetic Performance of a Novel Stator Modular Ring Drive Thruster Motor. Energies 2018, 11, 1598. [CrossRef]

17. Butler, N.A. Optimal and orthogonal Latin hypercube designs for computer experiments. Biometrika 2001, 88, 847-857. [CrossRef]

18. Deb, K. A Fast and Elitist Multiobjective Genetic Algorithm: NSGA-II. IEEE Trans. Evol. Comput. 2002, 6, 182-197. [CrossRef]

19. Horiuchi, T. Demagnetization Function; ANSYS Korea: Seoul, Korea, 2016.

20. Chau, K.T. Electric Vehicle Machines and Drives: Design, Analysis and Application, 1st ed.; Wiley-IEEE: Hoboken, NJ, USA, $2015 ;$ p. 243.

21. PIDOTECH Inc. PIAnO User's Manuals and Tutorials; PIDOTECH Inc.: Seoul, Korea, 2019.

22. You, Y.-M. Multi-Objective Optimal Design of Permanent Magnet Synchronous Motor for Electric Vehicle Based on Deep Learning. Appl. Sci. 2020, 10, 482. [CrossRef] 